\title{
Band Alignment and Charge Transfer in Complex Oxide Interfaces
}

\author{
Zhicheng Zhong ${ }^{1}$ and Philipp Hansmann ${ }^{1,2}$ \\ ${ }^{1}$ Max-Planck-Institut für Festkörperforschung, Heisenbergstrasse 1, 70569 Stuttgart, Germany \\ ${ }^{2}$ Institut für Theoretische Physik, Eberhard Karls Universität Tübingen, \\ Auf der Morgenstelle 14, 72076 Tübingen \\ (Received 4 July 2016; published 3 March 2017)
}

\begin{abstract}
The synthesis of transition metal heterostructures is currently one of the most vivid fields in the design of novel functional materials. In this paper, we propose a simple scheme to predict band alignment and charge transfer in complex oxide interfaces. For semiconductor heterostructures, band-alignment rules like the well-known Anderson or Schottky-Mott rule are based on comparison of the work function or electron affinity of the bulk components. This scheme breaks down for oxides because of the invalidity of a single work-function approximation as recently shown in [Phys. Rev. B 93, 235116 (2016); Adv. Funct. Mater. 26, 5471 (2016)]. Here, we propose a new scheme that is built on a continuity condition of valence states originating in the compounds' shared network of oxygen. It allows for the prediction of sign and relative amplitude of the intrinsic charge transfer, taking as input only information about the bulk properties of the components. We support our claims by numerical density functional theory simulations as well as (where available) experimental evidence. Specific applications include (i) controlled doping of $\mathrm{SrTiO}_{3}$ layers with the use of $4 d$ and $5 d$ transition metal oxides and (ii) the control of magnetic ordering in manganites through tuned charge transfer.
\end{abstract}

DOI: 10.1103/PhysRevX.7.011023

Subject Areas: Computational Physics,

Condensed Matter Physics,

Materials Science

\section{INTRODUCTION}

Until now, semiconductors present the most important class of functional materials for electronic applications. Their usage in electronic components and the continuous development of new devices keeps pushing the limits of technology. In almost all such devices, the key functionality originates not in the physics of the bulk but in the peculiarities of interfaces [1]. Yet, semiconductor devices have intrinsic limitations: (i) the characteristic length scales are relatively large so further downscaling (current state of the art is 7-nm technology) becomes very unlikely and Moore's law is bound to end; (ii) solely charge degrees of freedom are exploited. Transition metal oxides (TMO), on the other hand, provide spin, orbital, charge, and lattice degrees of freedom $[2,3]$ and are therefore viewed as one of the best candidates to replace semiconductors in future electronic devices. Thanks to the immense progress of epitaxial growth techniques, TMO heterostructures can now be controlled on atomic length scales. Several novel physical phenomena have been discovered in recent years, and potential multifunctional devices seem to be realizable [4-8].

Published by the American Physical Society under the terms of the Creative Commons Attribution 3.0 License. Further distribution of this work must maintain attribution to the author(s) and the published article's title, journal citation, and DOI.
In oxide electronics, one of the cornerstone mechanisms in complex heterostructures (in analogy to semiconductors) is the alignment of bands at a heterointerface and, driven by the resulting potential gradient, a charge transfer across the interface [9-28]. This mechanism can be seen as intrinsic doping without the undesired disorder induced by chemical doping. Consequently, controlling heterostructures with a wide variety and range of experimentally tunable parameters (e.g., strain, thickness, substrate choice, etc.) allows us to engineer new phases that do not exist in the bulk. Hence, it is obvious that the predictive power for the direction and amplitude of charge transfer with rules that are as simple as possible is highly desirable.

A natural first attempt would be the usage of wellestablished semiconductor rules, such as Anderson's or the Schottky-Mott rule [29]. As schematically shown in Fig. 1(a), the vacuum energy levels of the two semiconductors, which rely on their respective electron affinity (or work function), on either side of the heterojunction should be aligned. However, those rules are not suitable for TM oxides, due to the fact that the reduction of the work function to a single value is an approximation that does not hold for TM oxides [30,31]; for instance, for $\mathrm{SrTiO}_{3}$, the work function of a SrO-terminated (001) surface is $2.5 \mathrm{eV}$, while that of a $\mathrm{TiO}_{2}$-terminated surface is $4.2 \mathrm{eV}$ [32]. Moreover, the characteristic length scales in oxides are 1 or 2 orders of magnitude smaller than in semiconductors; hence, certain approximations are no longer justified, so nontrivial microscopic terms need to be taken into account explicitly. 
(a) Band alignment of semiconductor heterojunction
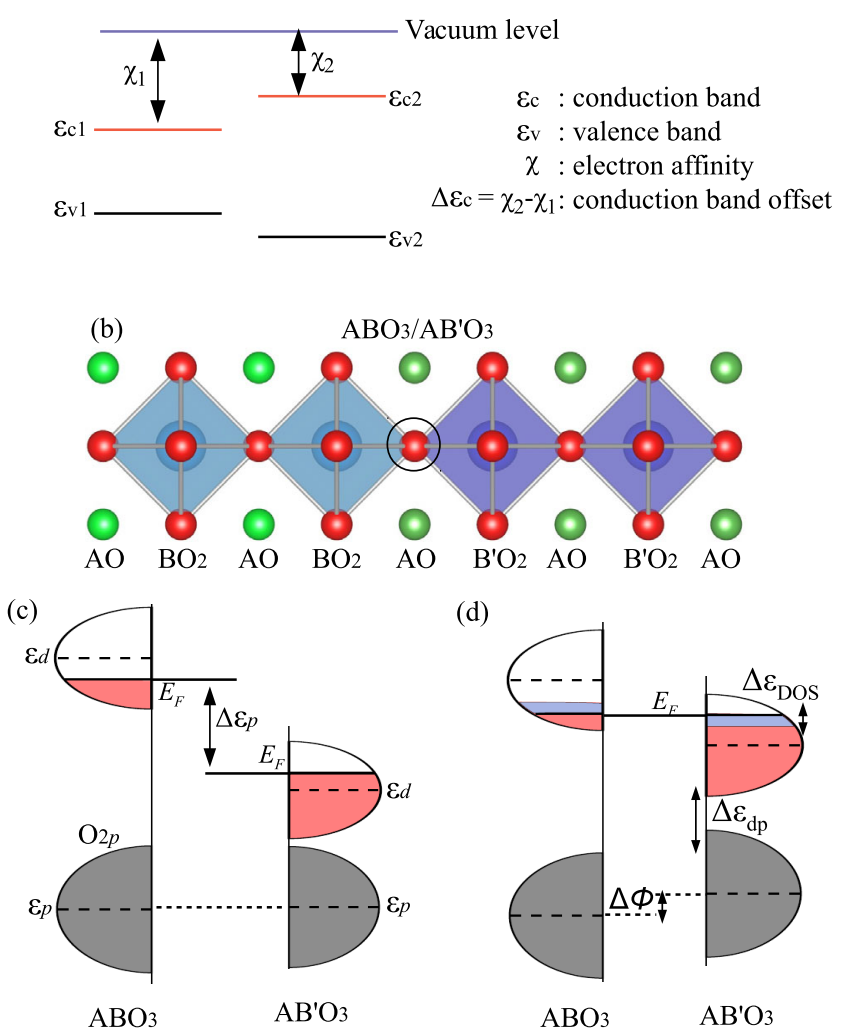

(d)

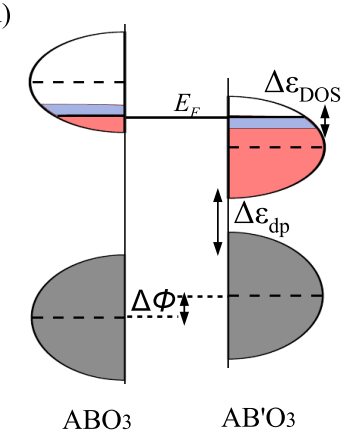

FIG. 1. (a) Schematic figure of Anderson's rule in semiconductor heterojunctions: The vacuum energy levels of the two semiconductors on either side are aligned. (b) Schematic figure of a perovskite (001) interface $\mathrm{ABO}_{3} / \mathrm{AB}^{\prime} \mathrm{O}_{3}$ emphasizing the common network of oxygen sites across the interface. (c) Alignment of oxygen states at the interface would generally yield a mismatch in the heterostructure's Fermi energy [see Eq. (1)]. This mismatch drives a charge transfer, which itself alters interface and local potentials. The final equilibrium state is shown in panel (d), with indications (black arrows) of the three different contributions in the energy balance equation [Eq. (2)]. Note that $\varepsilon_{p}$ and $\varepsilon_{d}$ are the local energy levels of oxygen $p$ and TM $d$ states.

In this paper, we propose a rule that is based on the continuity of states in the TMO's oxygen matrix and that allows for a qualitative prediction of band alignments and charge transfer in complex TMO heterostructures. The oxygen continuity boundary condition allows us to explain and predict the induced charge transfer between the constituents of the heterostructure starting only from energetics of the bulk compounds. In the first part of the paper, we sketch the underlying driving forces in TMO heterocompounds, which are built from perovskites $\mathrm{ABO}_{3}$, with A being a cation (e.g., Sr or La) and B a TM from a correlated $3 d$ or $4 d$ shell, or strongly spin-orbit coupled $5 d$ shell. We claim that bulk data for the oxygen $2 p$ energies $\varepsilon_{p}$ of the components can be used for predictions of $\mathrm{ABO}_{3} / \mathrm{AB}^{\prime} \mathrm{O}_{3}$ interfaces, and we prove this by showing quantitative data for a wide range of materials. In the second part of the paper, we provide selected examples as a proof of principle, as well as predictions for possible devices yet to be synthesized.

\section{METHODS}

To reveal general material trends, we first study the bulk transition metal oxides $\mathrm{ABO}_{3}$ with a cubic perovskite structure (in the majority of cases, A is taken as $\mathrm{Sr}$, but we also discuss other cases like $\mathrm{Ca}$ and $\mathrm{La}$ ), with $\mathrm{B}$ being a $3 d$ (Ti-Co), $4 d$ (Zr-Rh), or $5 d$ (Hf-Ir) transition metal element. The lattice constant is fixed at the optimized value of $\mathrm{SrTiO}_{3}, a=3.945 \AA$, which is $1 \%$ larger than the experimental value $a=3.905 \AA$. The $1 \%$ compressive strain has a general tendency to lower the energy levels of oxygen $p$ states by $0.1-0.2 \mathrm{eV}$ in all $\mathrm{ABO}_{3}$. We then use $\mathrm{SrRuO}_{3}$ as an example for effects of strain, cation A substitution, structural distortion, and magnetism. Most calculations were carried out for superlattices of $\left(\mathrm{ABO}_{3}\right)_{n} /\left(\mathrm{AB}^{\prime} \mathrm{O}_{3}\right)_{n}$ with $n=5$ and $n=1$, in order to estimate possible quantum confinement effects for the latter. Moreover, in order to validate the assumption of clean interfaces, we performed simulations of a rough interface with a $25 \%$ cation mixture (for details, see Appendix B). It turns out that the observed changes in the electronic structure are indeed small and will not affect our conclusions. For the study of magnetism in $\mathrm{SrMnO}_{3}$ heterostructures, we take into account an on-site Coulomb interaction $\mathrm{U}_{M n}=2 \mathrm{eV}$ and a realistic $\mathrm{GdFeO}_{3}$-type structural distortion, which are considered to be important for a realistic description of the magnetism. In all calculations, atomic positions are fully relaxed.

Our density functional theory (DFT) calculations were performed with the VASP (Vienna $a b$ initio simulation package) code [33] using the generalized gradient approximation (GGA) functional [34] for electronic exchange and correlation. We consider that energy separation between transition metal $d$ states and oxygen $2 p$ states is usually underestimated in the GGA potential, and we use the modified Becke Johnson (MBJ) potential [35], which is implemented in WIEN2k [36]. We perform Wannier projection $[37,38]$ of the oxygen $p$ Bloch states and TM $t_{2 g} d$ states to obtain an accurate value of the local energy levels $\varepsilon_{p}$ and $\varepsilon_{d}$ [39] as shown in Fig. 4 and Table I. Note that $\varepsilon_{p}$ and $\varepsilon_{d}$ correspond to local energy terms of $t_{2 g}$ and oxygen $p$ states, respectively, and not to the center of gravity for valence or conduction bands $\left(\varepsilon_{v}\right.$ and $\left.\varepsilon_{c}\right)$ [40]. Here, $\varepsilon_{p}$ and $\varepsilon_{v}$ coincide only in the ionic limit, but for finite covalence (i.e., oxygen-TM hybridization), $\varepsilon_{v}$ and $\varepsilon_{c}$ are split apart, and the bands no longer have pure oxygen or TM character. The values in Fig. 4 correspond to $\varepsilon_{d}$ and $\varepsilon_{p}$. It turns out that covalence yields an energy drop of approximately $0.4 \mathrm{eV}$ of $\varepsilon_{p}$ for all materials listed in Fig. 4. We have also carefully checked that the projection details, such as including $e_{g}$ orbitals, will not change our conclusions. 
TABLE I. Sign and trends of electron transfer in $\left(\mathrm{SrBO}_{3}\right)_{1} /$ $\left(\mathrm{SrB}^{\prime} \mathrm{O}_{3}\right)_{1}$ heterostructures. We list the energies of oxygen $2 p$ states resolved by $\mathrm{BO}_{2}\left[\varepsilon_{p}^{i}\left(\mathrm{BO}_{2}\right)\right], \mathrm{AO}\left[\varepsilon_{p}^{i}(\mathrm{AO})\right]$, and $\mathrm{B}^{\prime} \mathrm{O}_{2}$ layers $\left[\varepsilon_{p}^{i}\left(\mathrm{~B}^{\prime} \mathrm{O}_{2}\right)\right]$ with respect to the Fermi energy, as well as the induced electron transfer $\Delta n_{e}$ from $\mathrm{B}$ to $\mathrm{B}^{\prime}$ sites. Note that $\Delta \varepsilon_{p}$, taken from Fig. 4, is also listed.

\begin{tabular}{lllllr}
\hline \hline $\mathrm{B} / \mathrm{B}^{\prime}$ & $\varepsilon_{p}^{i}\left(\mathrm{BO}_{2}\right)$ & $\varepsilon_{p}^{i}(\mathrm{AO})$ & $\varepsilon_{p}^{i}\left(\mathrm{~B}^{\prime} \mathrm{O}_{2}\right)$ & $\Delta n_{e}$ & $\Delta \varepsilon_{p}$ \\
\hline $\mathrm{Ti} / \mathrm{Nb}$ & -4.67 & -4.93 & -5.22 & 0.36 & 2.03 \\
$\mathrm{Ti} / \mathrm{Ta}$ & -5.07 & -5.46 & -5.89 & 0.63 & 3.18 \\
$\mathrm{~V} / \mathrm{Nb}$ & -4.26 & -4.50 & -4.89 & 0.49 & 2.38 \\
$\mathrm{~V} / \mathrm{Ta}$ & -4.62 & -4.92 & -5.62 & 0.71 & 3.56 \\
$\mathrm{~V} / \mathrm{Cr}$ & -3.72 & -3.61 & -3.60 & -0.27 & -0.64 \\
$\mathrm{~V} / \mathrm{Mn}$ & -3.40 & -3.18 & -3.07 & -0.30 & -1.34 \\
$\mathrm{~V} / \mathrm{Fe}$ & -3.02 & -2.77 & -2.31 & -0.49 & -2.02 \\
$\mathrm{~V} / \mathrm{Co}$ & -2.65 & -2.42 & -1.38 & -0.57 & -2.43 \\
\hline \hline
\end{tabular}

In order to obtain quantitative estimates for the charge transfer, we integrate the electron density below the Fermi level (down to the energy gap between $d$ and oxygen $p$ states) projected inside the atomic spheres. The radii of the atomic spheres (here, we mention the ambiguity in the definition) were chosen as the default values of the projector augmented-wave (PAW) potential in VASP, e.g., $1.2 \AA$ for a vanadium atom in $\mathrm{SrVO}_{3}$. In this way, the integrated electron density of the vanadium atom in bulk $\mathrm{SrVO}_{3}$ is 0.75 . Considering the formal occupation of vanadium to be one $d$ electron, we then take 0.75 as a renormalizing factor, which we also apply to the interface case in order to obtain a quantitative value for the charge transfer. Moreover, to estimate the sensitivity of such values with respect to the choice of the radius of atomic spheres, we have checked that a $10 \%$ increase of the radius only induces approximately a 3\% change of the charge transfer estimated in this way. Hence, the numbers for the charge transfer given in Table I have to be considered with these definitions in mind. We stress that our conclusions do not rely on specific quantitative values but on overall trends.

\section{INTRINSIC CHARGE TRANSFER IN COMPLEX OXIDE INTERFACES}

Let us start the discussion with the idea that motivated the study. For the sake of simplicity, we restrict ourselves to perovskite $\mathrm{ABO}_{3}$ heterostructures along the (001) direction. As is common in such TMO compounds, the most relevant states are (i) the empty or partially filled TM $d$ states at the Fermi energy [41], which are split into $t_{2 g}$ and $e_{g}$ states in cubic perovskites, and (ii) the oxygen $2 p$ states residing at an energy $\varepsilon_{p}$ a few $\mathrm{eV}$ below the Fermi energy and forming more or less covalent bonds with the TM $d$ states. At an interface of two materials with different lattice constants or distortion patterns of an ideal cubic case, we typically find a smooth transition where such structural features mutually propagate between the components of the heterostructure $[42,43]$. A rather natural observation is that such smooth structural transitions in the shared oxygen lattice necessarily demand a continuity of the oxygen states across the interface layers as a boundary condition.

One might start by visualizing the oxygen continuity condition with a very simple sketch, which we show in Fig. 1(b) for an $\mathrm{ABO}_{3} / \mathrm{AB}^{\prime} \mathrm{O}_{3}$ interface; later, we extend our discussion to more general cases with different cations. In a hypothetical two-step procedure, the continuity condition would demand that, at the interface, the oxygen states need to be lined up, which, for two materials with different $\varepsilon_{p}$, would result in a mismatch of the Fermi energy $E_{F}$ equivalent to

$$
\Delta \varepsilon_{p}=\varepsilon_{p}^{\mathrm{ABO}_{3}}-\varepsilon_{p}^{\mathrm{AB}^{\prime} \mathrm{O}_{3}}
$$

[see Fig. 1(c)]. However, since $E_{F}$ must be constant all through the heterostructure in equilibrium, a charge transfer occurs between the layers, which itself creates (i) an electrostatic potential drop $\Delta \phi$ across the interface, (ii) rigid band shifts, which we indicate by $\Delta \varepsilon_{\text {DOS }}$, and (iii) a local electrostatic potential drop $\Delta \varepsilon_{d p}$ (with $\varepsilon_{d p}=\varepsilon_{d}-\varepsilon_{p}$ ) yielding relative shifts between TM $d$ and oxygen $p$. These three terms counter the original driving potential-see Fig. 1(d). As indicated in this last sketch, we end up with a balance of potentials that has to be calculated self-consistently. Before further formalization, let us support this hypothesis by numerical calculations starting with the easiest case of interfacing two band insulators, which, in fact, has a one-to-one correspondence with the band-alignment mechanism in semiconductor heterostructures.

In Fig. 2, we show the layer-dependent density of states for the interface of $\mathrm{SrTiO}_{3}$ and $\mathrm{SrZrO}_{3}$ simulated in a 5/5 superlattice. The chemical potential, which is basically free to move inside the gap, is indicated by the dashed black line, while the center of mass (i.e., the onsite energy) of the oxygen $2 p$ states (dark gray DOS) is shown as a black solid line. In the plots, we show $\mathrm{ZrO}_{2}$ and $\mathrm{TiO}_{2}$ layers directly at the interface, as well as the $\mathrm{TiO}_{2}$ bulklike layer two-unit cells further away from the interface. As can be seen, the alignment of oxygen states across the interface and even in the next $\mathrm{TiO}_{2}$ layer is practically perfect because of the absence of any charge transfer between the insulating layers. Please note that a similar alignment of oxygen states has also been reported in insulating $\mathrm{MgO}(111) / \mathrm{CaO}(111)$ interfaces with polar continuity [44]. This observation is a first indication of the validity of the backbone hypothesis of the oxygenstate continuity. We note in passing that we have an advantage compared to semiconductor heterostructures where obvious structural and electronic continuity is absent [45]. In such semiconducting heterojunctions, one might try to employ Anderson's (or the SchottkyMott) rules in order to estimate the band mismatch by the 
(a)

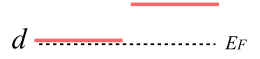

$\mathrm{O}_{p} \overline{\mathrm{SrTiO}_{3}} \overline{\mathrm{SrZrO}_{3}}$

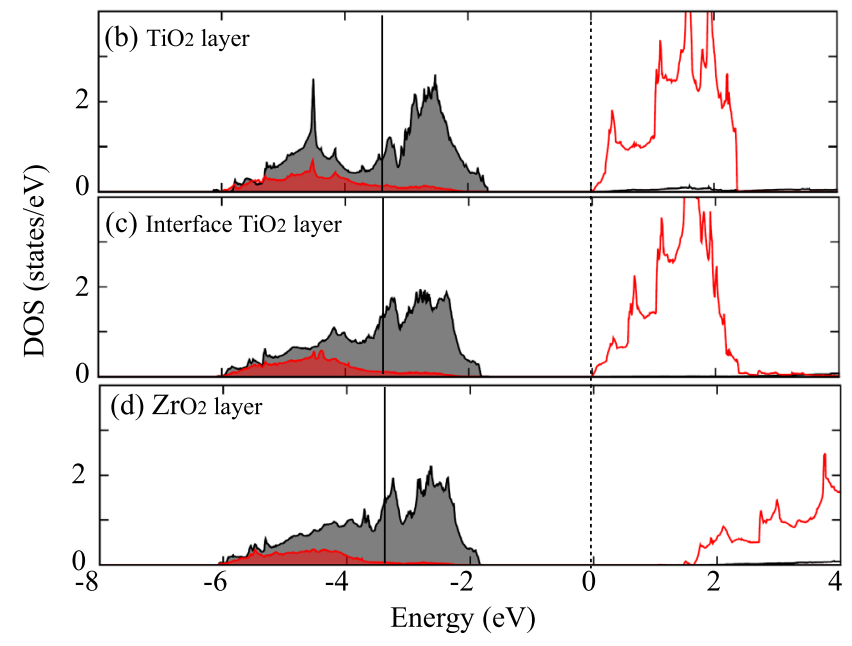

FIG. 2. (a) Sketch of the energy-level scheme, and (b)-(d) layer-resolved DFT density of states for the $\left(\mathrm{SrTiO}_{3}\right)_{5} /$ $\left(\mathrm{SrZrO}_{3}\right)_{5}$ superlattice. Oxygen $p$ states (dark gray) and TM $d$ states (red) are shown with the Fermi level marked by the dotted black line. Additionally, we show as a solid black line the layerdependent center of mass of the oxygen states, which corresponds to the layer-dependent $\varepsilon_{p}^{i}$. We plot the DOS for the two $\mathrm{BO}_{2}$ layers directly at the interface as well as for the $\mathrm{TiO}_{2}$ two-unit cell further away. The alignment of oxygen states in this bandinsulator heterostructure is practically perfect.

difference of the components' electron affinity (or work function) [29]. It turns out, however, that in realistic cases, these rules often fail to predict band offsets. Furthermore, a direct extrapolation of these rules to oxides is rather questionable due to the fact that the concept of a singlevalued work function is invalid as the current authors [30] and Jacobs et al. [31] have shown in recent studies of oxide heterostructure work functions, which strongly depend on details of the surface orientation and termination. In contrast, the oxygen-state continuity rule is a much stronger boundary condition and, as we point out in several occasions, its predictions seem to agree well with experimental observation.

Now, we take one step further and consider $\mathrm{SrNbO}_{3}$ instead of $\mathrm{SrZrO}_{3}$ in the same geometry, i.e., a metal with one valence electron in the $\mathrm{Nb} 4 d$ shell [46], which we show in Fig. 3-here, we see that an initial alignment of oxygen states has led to electron transfer from $\mathrm{Nb} 4 d$ to $\mathrm{Ti}$ $3 d$ states across the interface. The self-consistently determined final position of oxygen states with respect to the Fermi level is in agreement with the expectations from our sketch in Fig. 1(d). In other words, the transferred electrons lead to an additional potential $\Delta \phi$ in the interface region and changes in the local potentials (discussed in more detail (a)

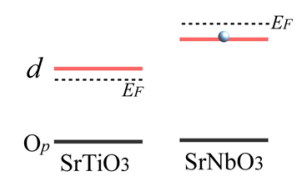

(b)

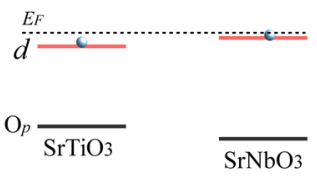

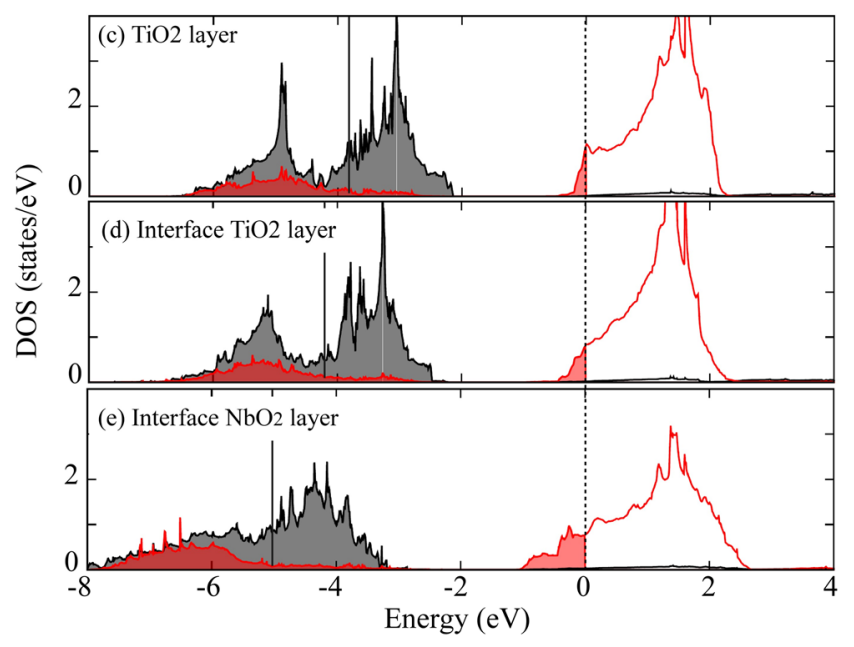

FIG. 3. Upper panel: Sketch of the energy-level scheme before (a) and after (b) electron transfer. Lower panels: Same plots as in Fig. 2 but now for the heterostructure $\left(\mathrm{SrTiO}_{3}\right)_{5} /\left(\mathrm{SrNbO}_{3}\right)_{5}$. Opposed to the $\left(\mathrm{SrTiO}_{3}\right)_{5} /\left(\mathrm{SrZrO}_{3}\right)_{5}$, we see a clear transfer of electrons across the interface and the expected structure in the alignment of the layer-dependent $\varepsilon_{p}^{i}$ in the layers shown. The electrons transferred into interfacial and bulk $\mathrm{TiO}_{2}$ layers are $0.23 e$ and $0.17 e$, respectively.

below), which eventually leads to a balanced monotonous evolution of the layer-dependent energy of oxygen $2 p$ states. Let us stress that, in our picture, the directions and relative strength of the charge transfer seem to be predictable just by comparison of $\Delta \varepsilon_{p}$ of the bulk $\mathrm{ABO}_{3}$.

Before providing numerical data and material calculations to confirm this, let us give more formal arguments. Indeed, the concept of band alignments at interfaces is well known and understood in semiconducting $p n$ junctions [47], where one observes charge modulations in the so-called "space charge" region of the typical order of $\approx 100 \AA$. In the $p n$ junctions, two identical semiconductors are interfaced, which, however, have a Fermi-level mismatch due to different, either $p$ or $n$ type, kinds of doping, which is resolved by charge transfer. Yet, in our case, there are some crucial differences specific to oxide interfaces: First of all, our length scales are at least 1 order of magnitude smaller, and the variation of the induced potential on the order of a few $\AA$ clearly prohibits a semiclassical model commonly used for semiconductors. Second, we have to consider more microscopic details for the energy balance equation that finally determines $\Delta n_{e}$. Namely, in addition to $\Delta \phi$ (the only term of relevance for semiconductor $p n$ junctions), the charge transfer in oxides induces shifts in the local potentials of the different TM 
sites which might be disentangled into contributions (i) from mutual change of the valence of $\mathrm{B}$ and $\mathrm{B}^{\prime}$ site $\Delta \varepsilon_{d p}$, yielding relative shifts between TM $d$ and oxygen $2 p$ states with a sign equal to that of $\Delta n_{e}$, and (ii) from the specific structure of density of states $\Delta \varepsilon_{\text {DOs. }}$. In summary, we can write

$$
-\Delta \varepsilon_{p}=\Delta \phi+\Delta \varepsilon_{\mathrm{DOS}}+\Delta \varepsilon_{d p}
$$

The terms on the right-hand side depend on the transferred charge $\Delta n_{e}$, and we may try to linearize them. For the first term $\Delta \phi$, we can assume a plate capacitor model, which would yield a potential drop per unit cell of $\Delta \phi=\Delta n_{e} d / \epsilon$, with $d$ being the effective distance of charge transfer across the interface and $\epsilon$ the dielectric permittivity $[48,49]$. The second term can be simplified by assuming an approximately constant density of states around Fermi level $E_{F}$ to be $\Delta n_{e} D$, where $D=\left(1 / D_{B}\left(E_{F}\right)\right)+\left(1 / D_{B^{\prime}}\left(E_{F}\right)\right)$, with $D_{B}$ and $D_{B^{\prime}}$ the local density of states for $B$ and $B^{\prime}$ sites. This contribution $D$ can be seen from Fig. 3 to be of the order of up to $1 \mathrm{eV}$. The last term indicates the change of $\varepsilon_{d p}$ induced by the charge transfer that modifies the valence of transition metal. The argument for the linearity in $\Delta n_{e}$ of the last term is easily understood by considering a Hartree-type self-energy $\left(\propto n_{e}\right)$, so we can assume $\Delta \varepsilon_{d p} \approx \Delta n_{e} U_{H}$. $U_{H}$ reflects the change of the energy due to the static singleparticle mean-field energy that originates from electronic Coulomb interaction. Using a virtual crystal approximation for $\mathrm{SrVO}_{3}$ allows us to roughly estimate this contribution to be also of the order of $1 \mathrm{eV}$. Hence, assuming the charge transfer of the order of $\Delta n_{e}=1$ will lead, different from semiconductors, to non-negligible contributions of $D$ and $U_{H}$ since the first term $d / \epsilon$, i.e., the typical length scale, is much smaller in our oxide heterostructures than in semiconductors. We eventually arrive at a simplified linear relation between $\Delta n_{e}$ and $\varepsilon_{p}^{\text {bulk: }}$

$$
\Delta n_{e} \approx-\frac{1}{\left(d / \epsilon+D+U_{H}\right)} \Delta \varepsilon_{p}
$$

While the simplified equation is also hardly solvable in a closed form, it allows for a remarkable insight and confirmation of our initial idea: The sign and strength of the charge transfer at an interface between two materials, which turns out to be much larger than in semiconductor devices, should be determined by the difference of the respective bulk oxygen $2 p$ energies with respect to their Fermi level $\varepsilon_{p}$ [50].

With this insight, we generate DFT reference data for a variety of bulk compounds. The results are summarized in Fig. 4, where we plot the average energy of oxygen $2 p$ states (filled symbols) and the average energy of partially filled $d$ orbitals (empty symbols) with respect to the Fermi level [51] for $\mathrm{SrBO}_{3}$ (solid lines), with $\mathrm{B}$ being a $3 d$ (black),

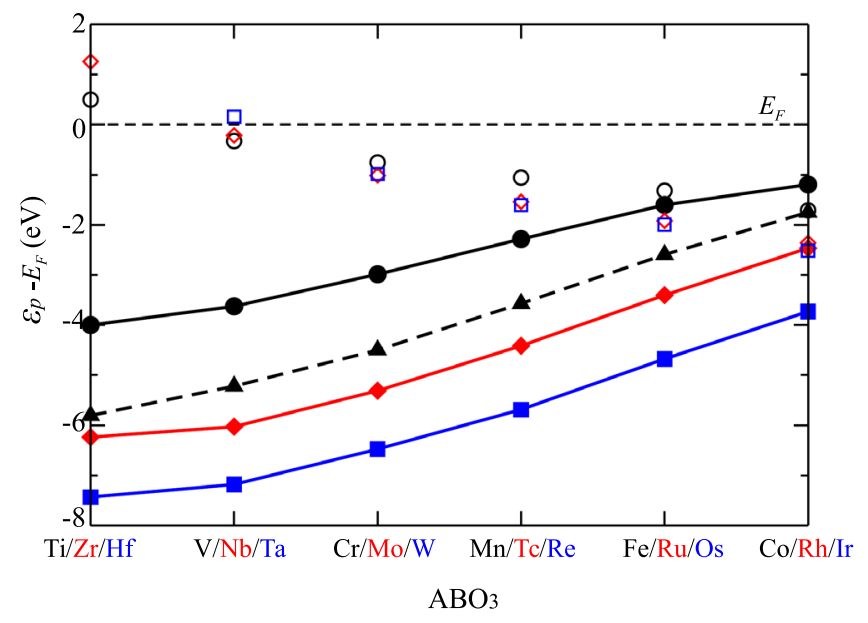

FIG. 4. Summary of bulk $\varepsilon_{p}$ (filled symbols) and $\varepsilon_{d}$ (empty symbols) with respect to the Fermi level $\left(E_{F}=0\right)$ for different $\mathrm{SrBO}_{3}$ (solid line) materials. For the $\mathrm{B}$ site, we consider $3 d$ (black), $4 d$ (red), and $5 d$ (blue) elements. With this reference bulk data, the charge transfer in heterostructures consisting of these materials can be anticipated. The simple criterion for the direction of the charge transfer at the $\mathrm{ABO}_{3} / \mathrm{AB}^{\prime} \mathrm{O}_{3}$ interface is that the component with lower $\varepsilon_{p}$ will donate electrons to the other one. Additionally, we plot data for $\mathrm{LaBO}_{3}$ (dashed line) for $\mathrm{B}=3 d$, which is used for estimates for $\mathrm{ABO}_{3} / \mathrm{A}^{\prime} \mathrm{BO}_{3}$ interfaces. (For numerical data of the plot and additional compounds, please refer to Table III in Appendix B.)

$4 d$ (red), or $5 d$ (blue) element. For the $3 d$ series, we additionally show oxygen $p$ energies for $\mathrm{LaBO}_{3}$ (dashed line). The figure shows clear trends in the series of materials considered:

(i) Within the intra $3 d, 4 d$, and $5 d$ series, we observe a monotonous and almost linear increase of $\varepsilon_{p}$ of about 2-3 eV within each period.

(ii) Within one group, we have a monotonous drop in $\varepsilon_{p}$ for a given configuration of about $\geq 3 \mathrm{eV}$ from $3 d$ to $5 d$ compounds.

(iii) Changing $\mathrm{Sr}$ to $\mathrm{La}$, i.e., decreasing the oxidation number of the transition metal, leads to a decrease of $\varepsilon_{p}$ up to $2 \mathrm{eV}$. This change is actually closely related to $\Delta \varepsilon_{d p}$ in Eq. (2) and represents, in some way, the extreme case where we have changed the nominal charge by 1 . Moreover, we note that a change of the cation with an identical oxidation state $(\{\mathrm{Sr}, \mathrm{Ca}, \mathrm{Ba}\}$ or $\{\mathrm{La}, \mathrm{Y}\}$ ) leaves the results for $\varepsilon_{p}$ basically unchanged (see Table IV in Appendix B).

Before we turn to the discussion of how these numbers can be used for predictions, we need to address the fact that the results shown in Fig. 4 are all obtained for undistorted cubic unit cells. The energy scale of the listed material trends is of the order of a few eV, and it should be emphasized that certain effects beyond idealized structures will lead to modifications of $\varepsilon_{p}$ on energy scales that are non-negligible compared to our reference data. Therefore, we have performed calculations for specific cases, studying the influence 
of strain, orthorhombic distortions, and magnetism (in Table IV in Appendix B, we provide the numerical data for these benchmark cases).

Starting with strain, we see, for the example of $\mathrm{SrRuO}_{3}$, that $1 \%$ compressive strain will decrease the $\varepsilon_{p}$ by $0.2 \mathrm{eV}$, and we realize that this effect can actually be additionally exploited to tune the energetics for the desired effect $[52,53]$. Next, we turn to orthorhombic distortions [22] in the same material for which we also observe a change of $\varepsilon_{p}$ of the order of $0.3 \mathrm{eV}$ compared to the cubic case, which is less but still important for a reliable prediction. Finally, let us address the influence of magnetic order for ferromagnetic (FM) $\mathrm{SrRuO}_{3}$. Here, we see a split between up and down states, not only in the Ru $4 d$ states but also in the associated oxygen states of $0.7 \mathrm{eV}$. We remark that this issue does not occur in antiferromagnetic (AFM)-ordered structures or for temperatures higher than the Curie temperature. In the FM-ordered phase, a prediction of the charge transfer by our simplified scheme is not straightforward, and it becomes questionable if the corresponding energy scales are equal to or larger than the $\Delta \varepsilon_{p}$ in question.

After these remarks, however, we now show that, for many cases, the numerical data for the bulk materials shown in Fig. 4 can be exploited in order to predict the charge transfer in layered heterostructures composed of the listed materials. Starting with the compounds shown in Figs. 2 and 3, i.e., $\mathrm{SrTiO}_{3}$ interfaces with $\mathrm{Sr}\{\mathrm{Zr}, \mathrm{Nb}\} \mathrm{O}_{3}$, we start with the bulk values $\varepsilon_{p}^{\mathrm{SrTiO}_{3}}=-4.0 \mathrm{eV}$ [54], $\varepsilon_{p}^{\mathrm{SrZrO}_{3}}=$ $-6.23 \mathrm{eV}$, and $\varepsilon_{p}^{\mathrm{SrNbO}_{3}}=-6.03 \mathrm{eV}$. For the $\mathrm{SrTiO}_{3} /$ $\mathrm{SrZrO}_{3}$ interface, both materials show empty TM $d$ states, and an alignment of $\varepsilon_{p}^{\mathrm{SrTiO}_{3}}$ with the lower-lying $\varepsilon_{p}^{\mathrm{SrZrO}_{3}}$ does not result in any electron transfer after equalizing the Fermi levels in line with Eq. (3) due to the diverging $D$ contribution in the right-hand-side denominator.

For the second case, however, we observe that, for $\mathrm{SrNbO}_{3}$, the oxygen states are much lower in energy than those of $\mathrm{SrTiO}_{3}$, i.e., $\Delta \varepsilon_{p}=2.03 \mathrm{eV} \gg 0$. Hence, an alignment of oxygen states would lead to a mismatch of the Fermi energy such that electrons need to be transferred from $\mathrm{Nb} 4 d$ states to the $\mathrm{Ti} 3 d$ states for a constant equilibrium chemical potential across the interface. We can exploit the above-reported trends in order to make general predictions:

(i) Given the monotonous trends within early materials of the same period in the $\mathrm{SrBO}_{3}$ series, we conclude that electron transfer will only be possible from lighter to heavier B compounds. This is somewhat counterintuitive: For instance, $d^{1} \mathrm{SrVO}_{3}$ will transfer electrons to $d^{2} \mathrm{SrCrO}_{3}$ but not to the empty Ti $3 d$ states of $\mathrm{SrTiO}_{3}$. As it turns out, this is in agreement with experiment and other numerical simulations for a variety of interfaces, including $\mathrm{LaMnO}_{3} / \mathrm{LaNiO}_{3} \quad[17,55-57], \quad \mathrm{LaTiO}_{3} / \mathrm{LaFeO}_{3}$ (a)

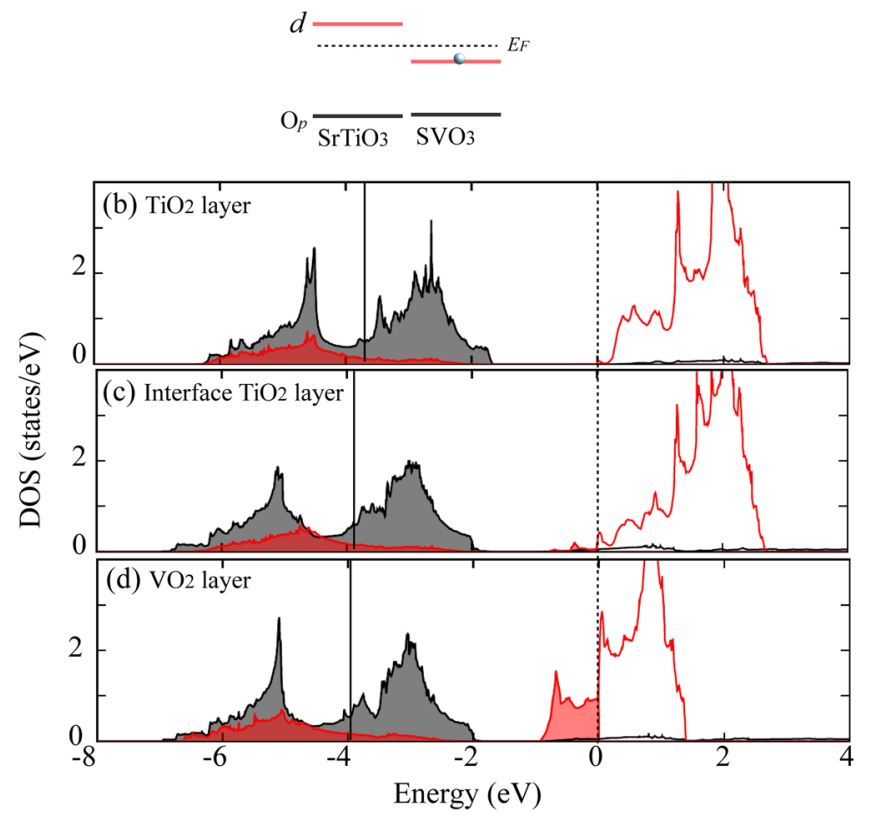

FIG. 5. Same plot as in Figs. 2 and 3 for a $\left(\mathrm{SrTiO}_{3}\right)_{5} /\left(\mathrm{SrVO}_{3}\right)_{5}$ heterostructure. In this case, alignment of oxygen bands will not drive electron transfer, and (as expected from Fig. 4) $\mathrm{SrVO}_{3}$ is a bad candidate for doping $\mathrm{SrTiO}_{3}$

[19,58,59], $\mathrm{LaTiO}_{3} / \mathrm{LaNiO}_{3}[18,23]$, and $\mathrm{SrVO}_{3} /$ $\mathrm{SrMnO}_{3}[52,53]$.

(ii) Given the monotonous behavior of $\varepsilon_{p}$ within one group over different periods, we can state that here electrons will also be transferred from the heavier to the lighter B elements; e.g., $\mathrm{SrNbO}_{3}$ will electron dope $\mathrm{SrVO}_{3}$.

(iii) For the more general case of $\mathrm{ABO}_{3} / \mathrm{AB}^{\prime} \mathrm{O}_{3}$, one has to consider precise values to make predictions. For instance, $\mathrm{SrIrO}_{3}$ should be able to intrinsically dope $\mathrm{SrMnO}_{3}$ at an interface, while $\mathrm{SrRuO}_{3}$ and $\mathrm{SrIrO}_{3}$ [60] cannot dope $\mathrm{SrTiO}_{3}$.

In order to strengthen the first claim of this list, we extend our simulations with a calculation of a $\mathrm{SrTiO}_{3} /$ $\mathrm{SrVO}_{3}$ interface for which we find $\Delta \varepsilon_{p}=-0.37 \mathrm{eV}$. Indeed, consistent with our arguments, we see from Fig. 5 that practically no charge transfer occurs. The layer-resolved partial density of states shows that basically all $d$ electrons remain in the $\mathrm{SrVO}_{3}$ slab, and the oxygen $2 p$ states are rather well aligned [61].

At this point, we might ask if our analysis can also be applied to periodic stacks that are further away from the limit of a single interface than the $5 / 5$ stacks considered so far. In order to answer this question, we performed several simulations of $\left(\mathrm{SrBO}_{3}\right)_{1} /\left(\mathrm{SrB}^{\prime} \mathrm{O}_{3}\right)_{1}$ heterostructures and summarize the results in Table I. Remarkably, we find that in all cases, the charge transfer can be anticipated in direction and relative amplitude with the data from Fig. 4. This is remarkable since it is not a priori clear 
that a qualitative argument made for the interface between two bulk materials still holds for a periodic stack with potentially strong quantum confinement effects [62] and lattice relaxations at the interfaces. They are fully taken into account in the heterostructure calculations but do not spoil the predictive capabilities from bulk calculations either. Looking more closely at the values in Table I reveals a remarkable consistency between $\Delta \varepsilon_{p}$ and $\Delta n_{e}$. We have already seen, in the example of the $\mathrm{SrTiO}_{3} / \mathrm{SrVO}_{3}$ interface, that in order to dope electrons into Ti or V $3 d$ states, $4 d$ or $5 d$ TM components have to be considered since a positive $\Delta \varepsilon_{p}$ is needed and, as we can see from the stacks in the first four rows, $\Delta n_{e}$ grows monotonously with $\Delta \varepsilon_{p}$. The same is true for the opposite case (reported in the lower four rows), where an increased negative $\Delta \varepsilon_{p}$ drives an increased transfer from the vanadium $3 d$ states to heavier elements like $\mathrm{Cr}, \mathrm{Mn}, \mathrm{Fe}$, and $\mathrm{Co}$ in the $3 d$ series. Moreover, we can learn from the observation $\Delta \varepsilon_{p}>\varepsilon_{p}^{i}(\mathrm{~B})-\varepsilon_{p}^{i}\left(\mathrm{~B}^{\prime}\right)$ that, for the energy balance [Eq. (2)] besides $\Delta \phi, \Delta \varepsilon_{\text {DOS }}$ and $\Delta \varepsilon_{\mathrm{dp}}$ contribute significantly.

Before the end of this section, where we have focused mostly on interfaces of the form $\mathrm{SrBO}_{3} / \mathrm{SrB}^{\prime} \mathrm{O}_{3}$, let us make some remarks about the generalization to $\mathrm{ABO}_{3} /$ $\mathrm{A}^{\prime} \mathrm{BO}_{3}$ and the most general $\mathrm{ABO}_{3} / \mathrm{A}^{\prime} \mathrm{B}^{\prime} \mathrm{O}_{3}$ interfaces. In Fig. 4, we have already shown data for $\mathrm{LaBO}_{3}$ in comparison to the $\mathrm{SrBO}_{3}$ series. If we consider the same element on the B site, our scheme is directly applicable as before. More specifically, $\varepsilon_{p}$ of a $\mathrm{LaBO}_{3}$ perovskite is always lower than in the corresponding $\mathrm{SrBO}_{3}$, so at a $\mathrm{LaBO}_{3} / \mathrm{SrBO}_{3}$ interface, the electrons are always transferred from $\mathrm{LaBO}_{3}$ to $\mathrm{SrBO}_{3}$, which is consistent with experiments, e.g., for the case of $\mathrm{LaTiO}_{3} / \mathrm{SrTiO}_{3}[9,63]$ or $\mathrm{LaMnO}_{3} / \mathrm{SrMnO}_{3}[13,16]$ superlattices with a symmetric slab configuration.

Additional complications arise in the most general $\mathrm{ABO}_{3} / \mathrm{A}^{\prime} \mathrm{B}^{\prime} \mathrm{O}_{3}$ setup with two different interfaces, $\mathrm{AO}-\mathrm{B}^{\prime} \mathrm{O}_{2}$ or $\mathrm{BO}_{2}-\mathrm{A}^{\prime} \mathrm{O}$, with polar discontinuity. A polar catastrophe will appear (see, e.g., the comprehensive review about polar oxide surfaces [64] and the particular case of $\left.\mathrm{LaAlO}_{3} / \mathrm{SrTiO}_{3}[26,48,49]\right)$. Polar discontinuity is a delicate issue and adds nontrivial complexity, including, e.g., generation of oxygen vacancies and impurities. Nevertheless, experiments still suggest a very good alignment of oxygen $2 p$ valence states at those interfaces [26]. We therefore attempt to extend our model to analyze polar discontinuous interfaces such as $\mathrm{SrTiO}_{3} / \mathrm{LaMnO}_{3}$ $[14,15,24,65]$. Starting with the interface $\mathrm{TiO}_{2}-\mathrm{LaO}$, we assume a unit of $\mathrm{LaTiO}_{3}$ linked with $\mathrm{SrTiO}_{3}$ on the one side and $\mathrm{LaMnO}_{3}$ on the other-so we have broken the problem down to a $\mathrm{SrTiO}_{3} / \mathrm{LaTiO}_{3}$ and a $\mathrm{LaTiO}_{3} / \mathrm{LaMnO}_{3}$ interface, which we know how to handle. Similarly, the $\mathrm{SrO}-\mathrm{MnO}_{2}$ interface can be viewed as $\mathrm{SrMnO}_{3}$ linked with $\mathrm{SrTiO}_{3}$ on the one side and $\mathrm{LaMnO}_{3}$ on the other side. Therefore, we could expect transfer of electrons in the $\mathrm{TiO}_{2}-\mathrm{LaO}$ interface but not in the $\mathrm{SrO}-\mathrm{MnO}_{2}$ interface.
We conclude our first main section with the claim that charge transfer at the interfaces of perovskite heterostructures can be qualitatively predicted by comparison of bulk qualities.

\section{APPLICATIONS AND PREDICTIONS}

In the second part of our paper, we highlight two applications of our prediction scheme, starting with the controlled doping of $\mathrm{SrTiO}_{3}$ (see Sec. IV A), also drawing a parallel to heterogeneous semiconductor devices. We then turn to magnetic transitions in manganite heterostructures triggered by controlled charge transfer (see Sec. IV B), explaining past theoretical and experimental results in the context of our unifying concept, which we then complement by predictions on how to improve external control of such transitions.

\section{A. Electron doping in $\mathrm{SrTiO}_{3}$ heterostructure}

As a first application, we chose to highlight how to control doping of one of the most widely used TMO compounds, $\mathrm{SrTiO}_{3}$. So far, $\mathrm{SrTiO}_{3}$ has been doped by various $3 d$ TM oxides, e.g., $\mathrm{LaAlO}_{3}$ [10] and $\mathrm{LaTiO}_{3}$ [9], to produce a two-dimensional electron gas, but there is no known $\mathrm{SrBO}_{3}$ with $\mathrm{B}$ from the $3 d$ series. With the data from Fig. 4, we have already given the reason why this is not surprising. The very naive guess that electrons from a more-filled $3 d$ element like, e.g., vanadium would spill into the empty Ti $3 d$ states is prohibited by the energy balance that originates in the oxygen-state continuity condition (see Fig. 5). Hence, $\mathrm{SrVO}_{3}$ is identified as a bad candidate for doping $\mathrm{SrTiO}_{3}$. Instead, we have seen in Fig. 3 that $\mathrm{SrNbO}_{3}$ is a much more promising component for that purpose. In $\left(\mathrm{SrTiO}_{3}\right)_{5} /\left(\mathrm{SrNbO}_{3}\right)_{5}$, the continuity of oxygen $2 p$ states drives a sizable charge transfer. What we have not discussed yet, however, is the question of whether microscopic details of orbital degrees of freedom at the interface, which are not included in our scheme, are equally or even more important for the charge transfer than the considered energetics. In order to shed light on this issue, we extend our calculations with the inclusion of an insulating buffer layer, which will allow for a spatial separation of $\mathrm{Ti}$ and $\mathrm{Nb}$ $d$ states. The choice of a perovskite buffer layer is actually quite straightforward in light of our reference data, and we chose $\mathrm{SrZrO}_{3}$ for this purpose, which will remain band insulating in the three-component heterostructure. In Fig. 6, we show the result for a symmetric $\left(\mathrm{SrTiO}_{3}\right)_{5} /\left(\mathrm{SrZrO}_{3}\right)_{2} /$ $\left(\mathrm{SrNbO}_{3}\right)_{1} /\left(\mathrm{SrZrO}_{3}\right)_{2}$ superlattice, which is experimentally realizable [21,66-68].

Here, we see another confirmation of our simple argument: While the buffer layer remains band insulating, we still observe a transfer of electrons from $\mathrm{Nb}$ to $\mathrm{Ti} d$ states even though they are spatially separated. At this point, we might refer to an analogous technique in semiconductor setups, where the doping impurities are spatially separated 
(a)

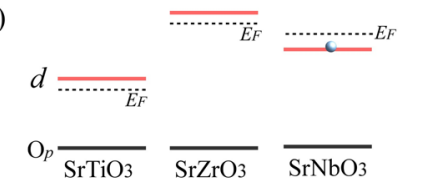

(b)

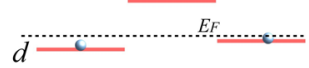

$\mathrm{O}_{p} \overline{\mathrm{SrTiO}_{3}} \overline{\mathrm{SrZrO}_{3}} \overline{\mathrm{SrNbO}_{3}}$
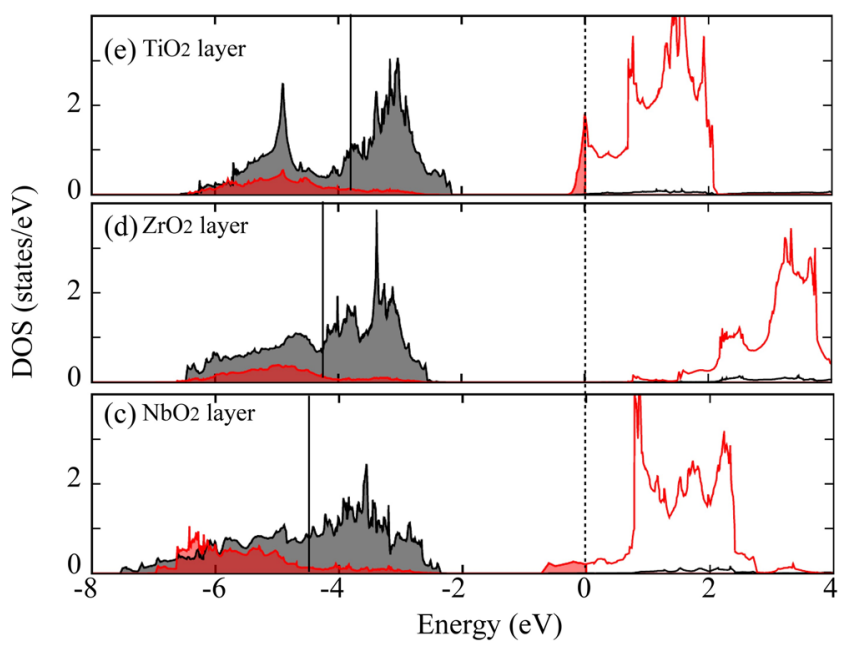

FIG. 6. Same plot as in Fig. 3 but now including an additional buffer layer between $\mathrm{SrTiO}_{3}$ and $\mathrm{SrNbO}_{3}$. The unit cell of this three-component symmetric heterostructure has the form $\left(\mathrm{SrTiO}_{3}\right)_{5} /\left(\mathrm{SrZrO}_{3}\right)_{2} /\left(\mathrm{SrNbO}_{3}\right)_{1} /\left(\mathrm{SrZrO}_{3}\right)_{2}$. As can be seen, the electron transfer from $\mathrm{Nb}$ to $\mathrm{Ti}$ remains intact even after inclusion of the buffer layer, excluding a pivotal role of microscopic details in the orbital degrees of freedom at the $\mathrm{SrTiO}_{3} /$ $\mathrm{SrNbO}_{3}$ interface.

from the charge carriers-a concept that is commonly known as "modulation doping" and widely applied in so-called MODFET setups [69,70]. Besides the results shown, we have checked the dependence of the charge transfer amplitude as a function of the thickness of the buffer layer but were unable to find significant trends up to supercells that include buffers of 5 bulk unit cells. In fact, modulation-doped oxides have recently started to attract some theoretical [49] and experimental [21,65,68] researchers.

\section{B. AFM-to-FM transition in $\mathrm{SrMnO}_{3}$ heterostructures}

After having exemplified how our oxygen energetics argument can be used to anticipate charge transfer in the case of $\mathrm{SrTiO}_{3}$-based heterostructures, we now take one step further by considering manganite heterostructures. Here, the goal is similar yet more ambitious since a provoked charge transfer in $\mathrm{SrMnO}_{3}$ setups should have a sensitive impact on magnetic transitions. Our starting point is bulk $\mathrm{SrMnO}_{3}$, which is found to have a G-type antiferromagnetic ground state. Our goal is to find an appropriate heterostructure in which doping into $\mathrm{Mn} d$ states triggers a transition to a ferromagnetic ground state-see review papers [71-74] and references therein.
TABLE II. Magnetic ground states of $\left(\mathrm{SrBO}_{3}\right)_{n} /\left(\mathrm{SrMnO}_{3}\right)_{n}$ with $n=1,2 . \mathrm{B}=\mathrm{Ir}, \mathrm{V}, \mathrm{Nb}$, and Ta. The magnetic moments localized on TM B and Mn sites are also listed.

\begin{tabular}{lcccccc}
\hline \hline & & $1 / 1$ & & & $2 / 2$ & \\
& $\mathrm{~B}\left(\mu_{B}\right)$ & $\mathrm{Mn}\left(\mu_{B}\right)$ & $\mathrm{M}$ & $B\left(\mu_{B}\right)$ & $\mathrm{Mn}\left(\mu_{B}\right)$ & $\mathrm{M}$ \\
\hline $\mathrm{SrIrO}_{3}$ & 0.0 & 3.24 & Ferro & 0.0 & 3.05 & AFM \\
$\mathrm{SrVO}_{3}$ & -0.19 & 3.2 & Ferri & -0.36 & 3.16 & Ferri \\
$\mathrm{SrNbO}_{3}$ & 0.05 & 3.66 & Ferro & 0.02 & 3.56 & Ferro \\
$\mathrm{SrTaO}_{3}$ & 0.03 & 3.74 & Ferro & 0.0 & 3.67 & Ferro \\
\hline \hline
\end{tabular}

From our reference data, we see that it should actually be rather easy to provoke transfer of electrons into $\mathrm{Mn} d$ states considering the comparatively large value of $\varepsilon_{p}$ for Mn. Indeed, it turns out that by employing our predictive scheme, we can confirm experimentally known routes to charge-transfer-induced magnetic transition and classify possible heterostructure components to tune more or less charge transfer.

We carried out calculations for $1 / 1$ and $2 / 2$ layered superlattice $\left(\mathrm{SrMnO}_{3}\right)_{1} /\left(\mathrm{SrBO}_{3}\right)_{1}$ (considering also a $\mathrm{GdFeO}_{3}$ structural distortion since magnetism can be strongly coupled to the lattice) for four different species of B site elements. The results are summarized in Table II, where we report the size of the magnetic moment on the B and the Mn site of the respective compound. We have sorted the table with ascending electron transfer. As anticipated, choosing $\mathrm{SrIrO}_{3}$ as an interfacing partner will result in little electron transfer, and in the $2 / 2$ structure, the antiferromagnetic order with a moment of $3.05 \mu_{B}$ remains. In the case of the $1 / 1$ structure, we observe a somewhat larger electron transfer, which is already sufficient to trigger a magnetic transition to a ferromagnetic ground state [25] with ordered Mn moments of $3.24 \mu_{B}$. The next component we consider is $\mathrm{SrVO}_{3}$-here, the data from Fig. 2 tell us to expect, similar to the Ir compound, only a small amount of transferred electrons, which we see confirmed in our numerical results. Interestingly, both $1 / 1$ and $2 / 2$ compounds seem to be intermediate between antiferromagnetic and ferromagnetic phases, yielding a ferrimagnetic ground state $[52,53]$. We can further predict which components would yield a much more significant electron transfer: Candidates are easily nominated from Fig. 4, and to this end, we select $\mathrm{SrNbO}_{3}$, which was recently grown experimentally [46], as well as $\mathrm{SrTaO}_{3}$ - our most potent electron donor for oxide heterostructures. The selected components perform as predicted in the numerical simulations, and we observe a large charge transfer pushing the $\left(\mathrm{SrMnO}_{3}\right)_{1,2} /\left(\mathrm{SrNbO}_{3}\right)_{1,2}$ and $\left(\mathrm{SrMnO}_{3}\right)_{1,2} /\left(\mathrm{SrTaO}_{3}\right)_{1,2}$ into a ferromagnetic metallic ground state with ordered Mn moments up to $3.74 \mu_{B}$ [75].

In order to underline the data given in Table II, we plot the resolved single-particle density of states in Fig. 7-this time, however, for magnetic DFT calculations. In the upper panels of the figure, we start, as a reference, with the bulk 


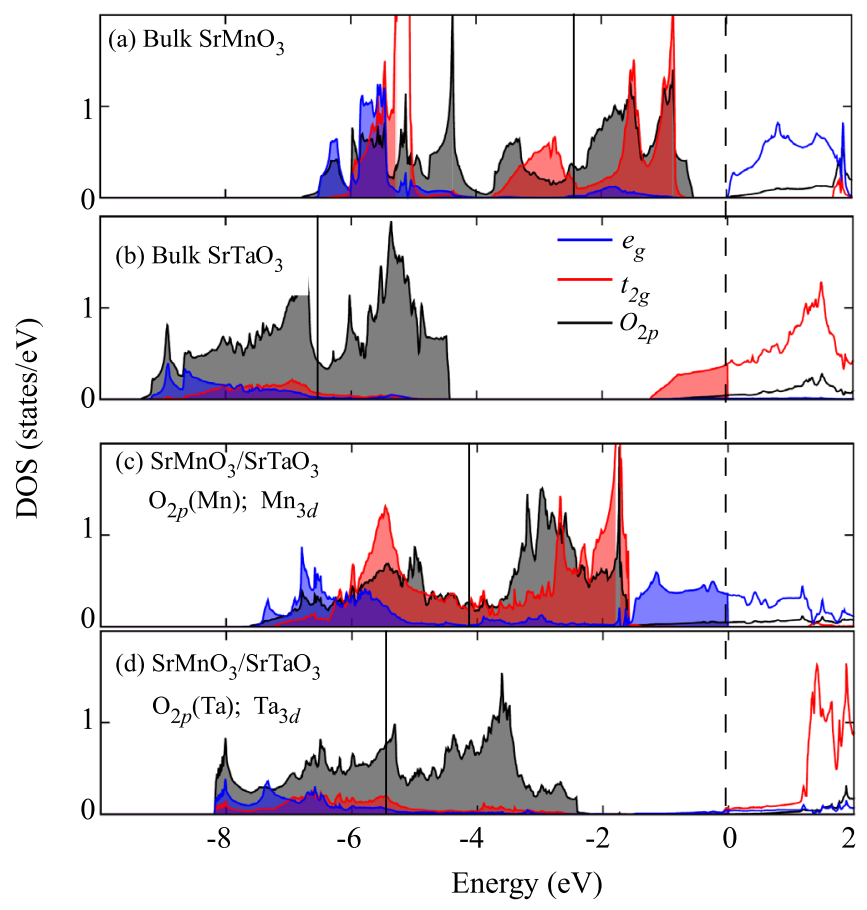

FIG. 7. Plot of the density of states with the usual color or marker convention (now also including $3 d-e_{g}$ states plotted in blue). We show results for magnetically ordered calculations, starting with bulk results in the upper two panels. $\mathrm{SrMnO}_{3}$ is G-type antiferromagnetic ordered and $\mathrm{SrTaO}_{3}$ paramagnetic. In the lower two panels, we plot the data for the two layers in a $\left(\mathrm{SrMnO}_{3}\right)_{1} /\left(\mathrm{SrTaO}_{3}\right)_{1}$ heterostructure, including a $\mathrm{GdFeO}_{3}$ distortion. We see the dramatic influence of the electron transfer from $\mathrm{Ta}$ to $\mathrm{Mn}$ and the induction of ferromagnetic-ordered metallic $\mathrm{MnO}_{2}$ layers.

results for the gapped antiferromagnetic DOS of $\mathrm{SrMnO}_{3}$ and metallic and paramagnetic $\mathrm{SrTaO}_{3}$. In the lower two panels, one can nicely observe how in the $\left(\mathrm{SrMnO}_{3}\right)_{1} /$ $\left(\mathrm{SrTaO}_{3}\right)_{1}$ heterostructure almost all Ta $d$ electrons are depleted in the $\mathrm{MnO}_{2}$ layers, yielding a metallic ferromagnetic ground state with significant filling of the Mn $3 d-e_{g}$ states.

\section{SUMMARY AND OUTLOOK}

In summary, we have presented a simple prediction scheme for the tuning of charge transfer in oxide heterostructures. By employing arguments based on the requirement that the $p$ states of the oxygen network in a heterostructure need to be continuous, we were able to utilize reference data from bulk calculations in Fig. 4 to predict the direction and relative amplitude of charge transfer between layers in oxide heterostructures. Remarkably, this scheme remains intact even for layered compounds down to a $1 / 1$ geometry. As a proof of principle, we provided simulations for electron doping of $\mathrm{SrTiO}_{3}$ in heterogeneous arrays, simultaneously confirming experimentally known trends and offering suggestions to material growers, which are the most promising components to control the low-energy electronic structure. In a second application part, we have exemplified, with the help of $\mathrm{SrMnO}_{3}$-based heterostructures, how controlled charge transfer can be systematically exploited in order to trigger magnetic phase transitions. Very recent experimental progress for $\mathrm{SrIrO}_{3} / \mathrm{SrMnO}_{3}$ confirms the predictions we made for the magnetic nature of the system [25]. The examples shown are only a taste of what can be predicted with the unifying concepts we presented, and we hope that experimental colleagues will be inspired by our work to create new materials and devices. This also includes, specifically, the possibility to couple or entangle-via charge transfer-physics of $3 d$ and $5 d$ TM oxides $[25,76]$, which, each on their own, are governed by very different energy scales. One might try, for instance, to induce spin-orbit coupling effects in $3 d$ systems, e.g., in the case of $\mathrm{SrMnO}_{3} / \mathrm{SrTaO}_{3}$ where one might find a large magnetic anisotropy energy and antisymmetric exchange on the magnetic Mn sites. One might even target the realization of yet-more-exotic Skyrmion, spin-spiral, and topological phases. On the computational side, we already started a hunt for the best possible candidates to be manipulated towards exciting new ground states.

In order to give an outlook for future theory and computational development, we state once more that for certain cases, the application of our scheme is not straightforward and it needs to be extended. One of these cases is the interfacing of compounds that are ferromagnetic ordered in the bulk. Another issue that should be mentioned is the change of $\varepsilon_{p}$ due to correlation effects: Given that the relevant effects we discussed have a characteristic energy scale of a few $\mathrm{eV}$, shifts due to single-particle self-energies might have to be included instead of the simplified $\Delta \varepsilon_{d p}$ contribution in Eq. (2). A very clear example where this will be the case is the usage of charge transfer insulators, typically found in late $3 d$ TM oxides, e.g., cuprates. An extension to include such correlation effects is very desirable since the sensitivity of correlated electron systems would add even more possibilities to generate novel functionality like, e.g., the recently suggested Mott transistor [77]. Moreover, focusing on material trends, the initial study presented did not extensively discuss how to use the thickness of periodic stacks or buffer layers to quantitatively tune charge transfer or how to interface nonperovskite oxides. The oxygen continuity condition, or adaptations thereof, should be valid not only in the complex oxides with perovskite structures but also in other lattice structures like anatase, pyrochlores, spinel, Ruddlesden-Popper, and double perovskites [78-80], which are structurally equal to the $1 / 1$ interfaces along a (111) direction.

\section{ACKNOWLEDGMENTS}

We thank H. Boschker, J. Nichols, H. N. Lee, Y. Lu, L. Si, Z. Liao, Z. Wang, Y. Cao, and J. Mannhart for motivation and valuable discussions. 


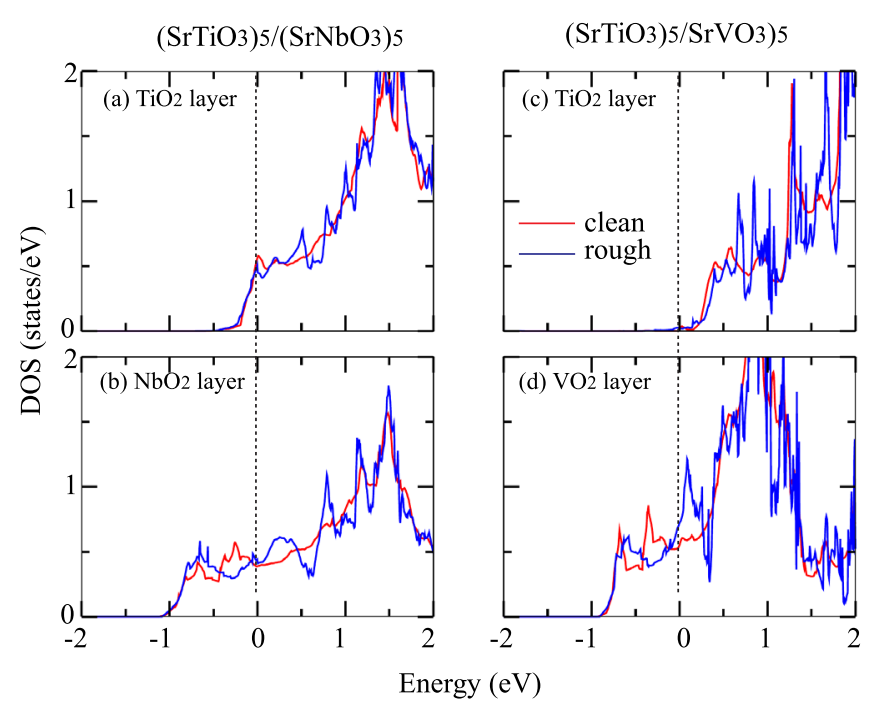

FIG. 8. Projected density of $\mathrm{TM} t_{2 g}$ states of the $\mathrm{BO}_{2}$ layer with two layers away from interface. Red indicates a clean interface, while blue indicates a rough interface with $25 \%$ cation intermixing.

\section{APPENDIX A: SIMULATION OF A ROUGH SURFACE}

To test the effect of cation mixing, we have performed benchmark calculations with up to $25 \%$ mixing. To this end, we employ a $2 \times 2$ supercell with a layered structure as $-\mathrm{BO}_{2}-\mathrm{AO}-\mathrm{B}_{0.75} \mathrm{~B}_{0.25}^{\prime} \mathrm{O}_{2}-\mathrm{AO}-\mathrm{B}_{0.25} \mathrm{~B}_{0.75}^{\prime} \mathrm{O}_{2}-\mathrm{AO}-\mathrm{B}^{\prime} \mathrm{O}_{2}$. The comparison between the density of states for clean and rough interfaces with (and without) charge transfer is shown in Fig. 8. Hence, our main conclusion remains unchanged, and effects like cation mixing can indeed be regarded as a secondary effect.

\section{APPENDIX B: NUMERICAL DATA}

In Table III, we provide the numerical data plotted in Fig. 4. Moreover, in Table IV, we report values of $\varepsilon_{p}$ for benchmark calculations, including effects of cation exchange, orthorhombic distortion, strain, and ferromagnetic ordering.

TABLE III. Numerical data of Fig. 4: $\varepsilon_{p}$ of $\mathrm{SrBO}_{3}$ with $\mathrm{B}=3 d, 4 d$, and $5 d$. The unit is $\mathrm{eV}$.

\begin{tabular}{lclclc}
\hline \hline $3 d$ & $\varepsilon_{p}$ & \multicolumn{1}{c}{$4 d$} & $\varepsilon_{p}$ & \multicolumn{1}{c}{$5 d$} & $\varepsilon_{p}$ \\
\hline $\mathrm{SrTiO}_{3}$ & -4.00 & $\mathrm{SrZrO}_{3}$ & -6.23 & $\mathrm{SrHfO}_{3}$ & -7.43 \\
$\mathrm{SrVO}_{3}$ & -3.63 & $\mathrm{SrNbO}_{3}$ & -6.03 & $\mathrm{SrTaO}_{3}$ & -7.18 \\
$\mathrm{SrCrO}_{3}$ & -2.99 & $\mathrm{SrMoO}_{3}$ & -5.31 & $\mathrm{SrWO}_{3}$ & -6.48 \\
$\mathrm{SrMnO}_{3}$ & -2.28 & $\mathrm{SrTcO}_{3}$ & -4.41 & $\mathrm{SrReO}_{3}$ & -5.69 \\
$\mathrm{SrFeO}_{3}$ & -1.60 & $\mathrm{SrRuO}_{3}$ & -3.40 & $\mathrm{SrOsO}_{3}$ & -4.68 \\
$\mathrm{SrCoO}_{3}$ & -1.19 & $\mathrm{SrRhO}_{3}$ & -2.47 & $\mathrm{SrIrO}_{3}$ & -3.73 \\
\hline \hline
\end{tabular}

TABLE IV. Reference data for $\varepsilon_{p}$ of manganites and ruthenates depending on cation exchange, orthorhombic distortion, compressive strain, and ferromagnetic ordering. The unit is $\mathrm{eV}$.

\begin{tabular}{lccc}
\hline \hline Manganites & \multicolumn{1}{c}{$\varepsilon_{p}$} & Ruthenates & $\varepsilon_{p}$ \\
\hline $\mathrm{KMnO}_{3}$ & -1.47 & $\mathrm{KRuO}_{3}$ & -2.48 \\
$\mathrm{CaMnO}_{3}$ & -2.30 & $\mathrm{CaRuO}_{3}$ & -3.44 \\
$\mathrm{BaMnO}_{3}$ & -2.22 & $\mathrm{BaRuO}_{3}$ & -3.29 \\
$\mathrm{LaMnO}_{3}$ & -3.57 & $\mathrm{LaRuO}_{3}$ & -4.56 \\
$\mathrm{SrMnO}_{3}$ & -2.28 & $\mathrm{SrRuO}_{3}$ & -3.40 \\
$1 \% \mathrm{compressive}_{\quad}^{\mathrm{SrMnO}}$ & -2.42 & $\mathrm{SrRuO}_{3}$ & -3.60 \\
Distorted CaMnO & & & \\
FM SrMnO & -2.70 & $\mathrm{SrRuO}_{3}$ & -3.72 \\
\hline \hline
\end{tabular}

[1] H. Kroemer, Nobel Lecture: Quasielectric Fields and Band Offsets: Teaching Electrons New Tricks, Rev. Mod. Phys. 73, 783 (2001).

[2] M. Imada, A. Fujimori, and Y. Tokura, Metal-Insulator Transitions, Rev. Mod. Phys. 70, 1039 (1998).

[3] Y. Tokura and N. Nagaosa, Orbital Physics in TransitionMetal Oxides, Science 288, 462 (2000).

[4] J. Mannhart and D. G. Schlom, Oxide Interfaces an Opportunity for Electronics, Science 327, 1607 (2010).

[5] H. Takagi and H. Y. Hwang, An Emergent Change of Phase for Electronics, Science 327, 1601 (2010).

[6] P. Zubko, S. Gariglio, M. Gabay, P. Ghosez, and J. M. Triscone, Interface Physics in Complex Oxide Heterostructures, Annu. Rev. Condens. Matter Phys. 2, 141 (2011).

[7] H. Y. Hwang, Y. Iwasa, M. Kawasaki, B. Keimer, N. Nagaosa, and Y. Tokura, Emergent Phenomena at Oxide Interfaces, Nat. Mat. 11, 103 (2012).

[8] J. Chakhalian, A. J. Millis, and J. Rondinel, Whither the Oxide Interface, Nat. Mat. 11, 92 (2012).

[9] A. Ohtomo, D. A. Muller, J. L. Grazul, and H. Y. Hwang, Artificial Charge-Modulation in Atomic-Scale Perovskite Titanate Superlattices, Nature 419, 378 (2002).

[10] A. Ohtomo and H. Y. Hwang, A High-Mobility Electron Gas at the $\mathrm{LaAlO}_{3} / \mathrm{SrTiO}_{3}$ Heterointerface, Nature 427, 423 (2004).

[11] I. González, S. Okamoto, S. Yunoki, A. Moreo, and E. Dagotto, Charge Transfer in Heterostructures of Strongly Correlated Materials, J. Phys. Condens. Mat. 20, 264002 (2008).

[12] G. Logvenov, A. Gozar, and I. Bozovic, High-Temperature Superconductivity in a Single Copper-Oxygen Plane, Science 326, 699 (2009).

[13] S. J. May, P. J. Ryan, J. L. Robertson, J.-W. Kim, T. S. Santos, E. Karapetrova, J. L. Zarestky, X. Zhai, S. G. E. te Velthuis, J. N. Eckstein, S. D. Bader, and A. Bhattacharya, Enhanced Ordering Temperatures in Antiferromagnetic Manganite Superlattices, Nat. Mat. 8, 892 (2009).

[14] J. Garcia-Barriocanal et al., Spin and Orbital Ti Magnetism at $\mathrm{LaMnO}_{3} / \mathrm{SrTiO}_{3}$ Interfaces, Nat. Commun. 10, 1038 (2010). 
[15] J. Garcia-Barriocanal, F. Y. Bruno, A. Rivera-Calzada, Z. Sefrioui, N. M. Nemes, M. Garcia-Hernández, J. RubioZuazo, G. R. Castro, M. Varela, S. J. Pennycook, C. Leon, and J. Santamaria, Charge Leakage at $\mathrm{LaMnO}_{3} / \mathrm{SrTiO}_{3}$ Interfaces, Adv. Mater. 22, 627 (2010).

[16] A. Bhattacharya, S. J. May, S. G. E. te Velthuis, M. Warusawithana, X. Zhai, Bin Jiang, J.-M. Zuo, M. R. Fitzsimmons, S. D. Bader, and J. N. Eckstein, MetalInsulator Transition and Its Relation to $\left(\mathrm{LaMnO}_{3}\right)_{2 \mathrm{n}} /$ $\left(\mathrm{SrMnO}_{3}\right)_{\mathrm{n}}$ Magnetic Structure in Superlattices, Phys. Rev. Lett. 100, 257203 (2008).

[17] M. Gibert, P. Zubko, R. Scherwitzl, J. Iniguez, and J. M. Triscone, Exchange Bias in $\mathrm{LaNiO}_{3}-\mathrm{LaMnO}_{3}$ Superlattice, Nat. Mat. 11, 195 (2009).

[18] H. Chen, A. J. Millis, and C. A. Marianetti, Engineering Correlation Effects via Artificially Designed Oxide Superlattices, Phys. Rev. Lett. 111, 116403 (2013).

[19] J. E. Kleibeuker, Z. Zhong, H. Nishikawa, J. Gabel, A. Müller, F. Pfaff, M. Sing, K. Held, R. Claessen, G. Koster, and G. Rijnders, Electronic Reconstruction at the Isopolar $\mathrm{LaTiO}_{3} / \mathrm{LaFeO}_{3}$ Interface: An X-Ray Photoemission and Density-Functional Theory Study, Phys. Rev. Lett. 113, 237402 (2014).

[20] J. Salafranca, J. Rincón, J. Tornos, C. León, J. Santamaria, E. Dagotto, S. J. Pennycook, and M. Varela, Competition between Covalent Bonding and Charge Transfer at Complex-Oxide Interfaces, Phys. Rev. Lett. 112, 196802 (2014).

[21] Y. Chen, F. Trier, T. Kasama, D. V. Christensen, N. Bovet, Z. I. Balogh, H. Li, K. T. S. Thydén, W. Zhang, S. Yazdi, P. Norby, N. Pryds, and S. Linderoth, Creation of High Mobility Two-Dimensional Electron Gases via Strain Induced Polarization at an Otherwise Nonpolar Complex Oxide Interface, Nano Lett. 15, 1849 (2015).

[22] M. N. Grisolia et al., Hybridization-Controlled Charge Transfer and Induced Magnetism at Correlated Oxide Interfaces, Nat. Phys. 12, 484 (2016).

[23] Y. Cao, X. Liu, M. Kareev, D. Choudhury, S. Middey, D. Meyers, J.-W. Kim, P. J. Ryan, J. W. Freeland, and J. Chakhalian, Engineered Mott Ground State in a $\mathrm{LaTiO}_{3} / \mathrm{LaNiO}_{3}$ Heterostructure, Nat. Commun. 7, 10418 (2016).

[24] X. R. Wang et al., Imaging and Control of Ferromagnetism in $\mathrm{LaMnO}_{3} / \mathrm{SrTiO}_{3}$ Heterostructures, Science 349, 716 (2015).

[25] J. Nichols et al., Emergence of Magnetism and Anomalous Hall Effect in Iridate-Manganite Heterostructures, Nat. Commun. 7, 12721 (2016).

[26] S. A. Chambers et al., Instability, Intermixing and Electronic Structure at the Epitaxial Heterojunction, Surf. Sci. Rep. 65, 317 (2010).

[27] S. A. Chambers, L. Qiao, T. C. Droubay, T. C. Kaspar, B. W. Arey, and P. V. Sushko, Band Alignment, Built-in Potential, and the Absence of Conductivity at the $\mathrm{LaCrO}_{3} /$ $\mathrm{SrTiO}_{3}(001)$ Heterojunction, Phys. Rev. Lett. 107, 206802 (2011).

[28] R. Comes and S. Chambers, Interface Structure, Band Alignment, and Built-in Potentials at $\mathrm{LaFeO}_{3} / \mathrm{n}-\mathrm{SrTiO}_{3}$ Heterojunctions, Phys. Rev. Lett. 117, 226802 (2016).
[29] J. H. Davies, The Physics of Low-Dimensional Semiconductors (Cambridge University Press, Cambridge, England, 1997).

[30] Z. Zhong and P. Hansmann, Tuning the Work Function in Transition Metal Oxides and Their Heterostructures, Phys. Rev. B 93, 235116 (2016).

[31] R. Jacobs, J. Booske, and D. Morgan, Understanding and Controlling the Work Function of Perovskite Oxides Using Density Functional Theory, Adv. Funct. Mater. 26, 5471 (2016).

[32] T. Susaki, A. Makishima, and H. Hosono, Work Function Engineering via $\mathrm{LaAlO}_{3} / \mathrm{SrTiO}_{3}$ Polar Interfaces, Phys. Rev. B 84, 115456 (2011).

[33] G. Kresse and D. Joubert, From Ultrasoft Pseudopotentials to the Projector Augmented-Wave Method, Phys. Rev. B 59, 1758 (1999).

[34] J. P. Perdew, K. Burke, and M. Ernzerhof, Generalized Gradient Approximation Made Simple, Phys. Rev. Lett. 77, 3865 (1996).

[35] F. Tran and P. Blaha, Accurate Band Gaps of Semiconductors and Insulators with a Semilocal Exchange-Correlation Potential, Phys. Rev. Lett. 102, 226401 (2009).

[36] P. Blaha, K. Schwarz, G. K. H. Madsen, D. Kvasnicka, and J. Luitz, WIEN2k, An Augmented Plane Wave + Local Orbitals Program for Calculating Crystal Properties (Karlheinz Schwarz, Techn. Universität Wien, Austria, 2001).

[37] J. Kuneš, R. Arita, P. Wissgott, A. Toschi, H. Ikeda, and K. Held, Wien2wannier: From Linearized Augmented Plane Waves to Maximally Localized Wannier Functions, Comput. Phys. Commun. 181, 1888 (2010).

[38] A. A. Mostofi, J. R. Yates, Y. S. Lee, I. Souza, D. Vanderbilt, and N. Marzari, Wannier90: A Tool for Obtaining Maximally-Localised Wannier Functions, Comput. Phys. Commun. 178, 685 (2008).

[39] M. W. Haverkort, M. Zwierzycki, and O. K. Andersen, Multiplet Ligand-Field Theory Using Wannier Orbitals, Phys. Rev. B 85, 165113 (2012).

[40] C. Noguera, Physics and Chemistry at Oxide Surfaces (Cambridge University Press, Cambridge, England, 1996).

[41] In band insulating materials such as $\mathrm{SrTiO}_{3}$, it turns out (from several DFT simulations of the material) that the assumption of a slight $n$-type doping ( $E_{F}$ at the bottom of the conduction band) is the appropriate choice.

[42] J. M. Rondinelli and N. A. Spaldin, Structure and Properties of Functional Oxide Thin Films: Insights from ElectronicStructure Calculations, Adv. Mater. 23, 3363 (2011).

[43] Z. Liao, M. Huijben, Z. Zhong, N. Gauquelin, S. Macke, R. J. Green, S. Van Aert, J. Verbeeck, G. Van Tendeloo, K. Held et al., Controlled Lateral Anisotropy in Correlated Manganite Heterostructures by Interface-Engineered Oxygen Octahedral Coupling, Nat. Mat. 15, 425 (2016).

[44] J. Goniakowski and C. Noguera, Conditions for Electronic Reconstruction at Stoichiometric Polar/Polar Interfaces, J. Phys. Condens. Mat. 26, 485010 (2014).

[45] J. Tersoff, Theory of Semiconductor Heterojunctions: The Role of Quantum Dipoles, Phys. Rev. B 30, 4874 (1984).

[46] D. Oka, Y. Hirose, S. Nakao, T. Fukumura, and T. Hasegawa, Intrinsic High Electrical Conductivity of 
Stoichiometric $\mathrm{SrNbo}_{3}$ Epitaxial Thin Films, Phys. Rev. B 92, 205102 (2015).

[47] N. W. Ashcroft and N. D. Mermin, Solid State Physics (Holt, Rinehart and Winston, Philadelphia, 1976).

[48] N. Nakagawa, H. Y. Hwang, and D. A. Muller, Why Some Interfaces Cannot Be Sharp, Nat. Mater. 5, 204 (2006).

[49] Z. Zhong, P.X. Xu, and P. J. Kelly, Polarity-Induced Oxygen Vacancies at $\mathrm{LaAlO}_{3} / \mathrm{SrTiO}_{3}$ Interfaces, Phys. Rev. B 82, 165127 (2010).

[50] Finally, let us remark that this relation also holds for the case of band-insulating interfaces (i.e., vanishing density of states at $E_{F}$ ), where $D$ will diverge, and hence an infinitesimal $\Delta n_{e}$ leads to finite $\Delta \varepsilon_{p}$. For a strongly correlated material, e.g., Mott insulator, a small change of charge density might lead to a significant change of $D$, and hence we could expect that the correlation effect will bring a new ingredient into our model.

[51] The change of $E_{F}$ within the series is quite small, e.g., $E_{F}\left(\mathrm{SrVO}_{3}\right)=5.20 \mathrm{eV}, E_{F}\left(\mathrm{SrMnO}_{3}\right)=5.13 \mathrm{eV}$.

[52] H. Chen, H. Park, A. J. Millis, and C. A. Marianetti, Charge Transfer across Transition-Metal Oxide Interfaces: Emergent Conductance and Electronic Structure, Phys. Rev. B 90, 245138 (2014).

[53] A. Tseng, A. Pham, S. C. Smith, and S. Li, Tuning Conductivity and Magnetism in Isopolar Oxide Superlattices via Compressive and Tensile Strain: A Case Study of $\mathrm{SrVO}_{3} / \mathrm{SrMnO}_{3}$ and $\mathrm{SrCrO}_{3} / \mathrm{SrMnO}_{3}$ Heterostructure, J. Appl. Phys. 119, 075301 (2016).

[54] For $\mathrm{SrTiO}_{3}$ with lattice constant $3.905 \AA, \varepsilon_{p}$ is $-4.11 \mathrm{eV}$.

[55] S. Dong and E. Dagotto, Quantum Confinement Induced Magnetism in $\mathrm{LaNiO}_{3}-\mathrm{LaMnO}_{3}$ Superlattices, Phys. Rev. B 87, 195116 (2013).

[56] A. T. Lee and M. J. Han, Charge Transfer, Confinement, and Ferromagnetism in $\mathrm{LaMnO}_{3}-\mathrm{LaNiO}_{3}$ (001) Superlattices, Phys. Rev. B 88, 035126 (2013).

[57] J. Hoffman, I. C. Tung, B. B. Nelson-Cheeseman, M. Liu, J. W. Freeland, and A. Bhattacharya, Charge Transfer and Interfacial Magnetism in $\left(\mathrm{LaNiO}_{3}\right)_{\mathrm{n}} /\left(\mathrm{LaMnO}_{3}\right)_{2}$ Superlattices, Phys. Rev. B 88, 144411 (2013).

[58] H. Zhang, Y. Weng, X. Yao, and S. Dong, Charge Transfer and Hybrid Ferroelectricity in $\left(\mathrm{YFeO}_{3}\right)_{\mathrm{n}} /\left(\mathrm{YTiO}_{3}\right)_{\mathrm{n}}$ Magnetic Superlattices, Phys. Rev. B 91, 195145 (2015).

[59] X. He and K. J. Jin, Engineering Charge Ordering into Multiferroicity, Phys. Rev. B 93, 161108 (2016).

[60] J. Matsuno, K. Ihara, S. Yamamura, H. Wadati, K. Ishii, V. V. Shankar, H.-Y. Kee, and H. Takagi, Engineering a SpinOrbital Magnetic Insulator by Tailoring Superlattices, Phys. Rev. Lett. 114, 247209 (2015).

[61] Taking a closer look, one should note a very small amount of charge at the interface $\mathrm{TiO}_{2}$ (concomitant with a nonperfect oxygen alignment) layer, which one might attribute to a covalent hybridization of $\mathrm{Ti}$ and $\mathrm{V} d$ states directly at the interface. However, as we will see in the next step, this effect is very small compared to the charge transfer one can obtain by replacing $\mathrm{SrVO}_{3}$ with another compound.

[62] Z. Zhong, Q. Zhang, and K. Held, Quantum Confinement in Perovskite Oxide Heterostructures: Tight Binding Instead of a Nearly Free Electron Picture, Phys. Rev. B 88, 125401 (2013).
[63] S. Okamoto and A. J. Millis, Electronic Reconstruction at an Interface between a Mott Insulator and a Band Insulator, Nature 428, 630 (2004).

[64] C. Noguera, Polar Oxide Surfaces, J. Phys. Condens. Matter 12, R367 (2000).

[65] Y. Z. Chen, F. Trier, T. Wijnands, R. J. Green, N. Gauquelin, R. Egoavil, D. V. Christensen, G. Koster, M. Huijben, N. Bovet et al., Extreme Mobility Enhancement of TwoDimensional Electron Gases at Oxide Interfaces by Charge-Transfer-Induced Modulation Doping, Nat. Mater. 14, 801 (2015).

[66] A. Beck, J. G. Bednorz, Ch. Gerber, C. Rossel, and D. Widmer, Reproducible Switching Effect in Thin Oxide Films for Memory Applications, Appl. Phys. Lett. 77, 139 (2000).

[67] M. L. Moreira, J. Andrés, V. M. Longo, M. S. Li, J. A. Varela, and E. Longo, Photoluminescent Behavior of $\mathrm{SrZrO}_{3} / \mathrm{SrTiO}_{3}$ Multilayer Thin Films, Chem. Phys. Lett. 473, 293 (2009).

[68] A. P. Kajdos, D. G. Ouellette, T. A. Cain, and S. Stemmer, Two-Dimensional Electron Gas in a Modulation-Doped $\mathrm{SrTiO}_{3} / \mathrm{Sr}(\mathrm{Ti}, \mathrm{Zr}) \mathrm{O}_{3}$ Heterostructure, Appl. Phys. Lett. 103, 082120 (2013).

[69] R. Dingle, H. L. Störmer, A. C. Gossard, and W. Wiegmann, Electron Mobilities in Modulation Doped Semiconductor Heterojunction Superlattices, Appl. Phys. Lett. 33, 665 (1978).

[70] T. Mimura, S. Hiyamizu, T. Fujii, and K. Nanbu, A New Field-Effect Transistor with Selectively Doped $\mathrm{GaAs} / \mathrm{n}-\mathrm{Al}_{\mathrm{x}} \mathrm{Ga}_{1-\mathrm{x}}$ As Heterojunctions, Jap. J. Appl. Phys. 19, L225 (1980).

[71] J. M. D. Coey, M. Viret, and S. von Molnr, Mixed-Valence Manganites, Adv. Phys. 48, 167 (1999).

[72] E. Dagotto, T. Hotta, and A. Moreo, Colossal Magnetoresistant Materials: The Key Role of Phase Separation, Phys. Rep. 344, 1 (2001).

[73] M. B. Salamon and M. Jaime, The Physics of Manganites: Structure and Transport, Rev. Mod. Phys. 73, 583 (2001).

[74] Y. Tokura, Critical Features of Colossal Magnetoresistive Manganites, Rep. Prog. Phys. 69, 797 (2006).

[75] Let us remark once more that, because of additional confinement effects, the $1 / 1$ compounds are the materials "furthest away" from a single interface situation, so some exceptions to the trends are quite expected-it is actually rather remarkable how well most of the predictions from bulk energetics still work.

[76] W. G. Yin, X. Liu, A. M. Tsvelik, M. P. M. Dean, M. H. Upton, J. Kim, D. Casa, A. Said, T. Gog, T. F. Qi, G. Cao, and J.P. Hill, Ferromagnetic Exchange Anisotropy from Antiferromagnetic Superexchange in the Mixed 3d-5d Transition-Metal Compound $\mathrm{Sr}_{3} \mathrm{CuIro}_{6}$, Phys. Rev. Lett. 111, 057202 (2013).

[77] Z. Zhong, M. Wallerberger, J. M. Tomczak, C. Taranto, N. Parragh, A. Toschi, G. Sangiovanni, and K. Held, Electronics with Correlated Oxides: $\mathrm{SrVO}_{3} / \mathrm{SrTiO}_{3}$ as a Mott Transistor, Phys. Rev. Lett. 114, 246401 (2015).

[78] Y. Tomioka, T. Okuda, Y. Okimoto, R. Kumai, K.-I. Kobayashi, and Y. Tokura, Magnetic and Electronic Properties of a Single Crystal of Ordered Double Perovskite $\mathrm{Sr}_{2} \mathrm{FeMoO}_{6}$, Phys. Rev. B 61, 422 (2000). 
[79] K. Kato, Y. Moritomo, M. Takata, M. Sakata, M. Umekawa, N. Hamada, S. Ohkoshi, H. Tokoro, and K. Hashimoto, Direct Observation of Charge Transfer in Double-Perovskite-like $\mathrm{RbMn}\left[\mathrm{Fe}(\mathrm{CN})_{6}\right]$, Phys. Rev. Lett. 91, 255502 (2003).
[80] Y. W. Long, N. Hayashi, T. Saito, M. Azuma, S. Muranaka, and Y. Shimakawa, Temperature-Induced $A B$ Intersite Charge Transfer in an A-Site-Ordered $\mathrm{LaCu}_{3} \mathrm{Fe}_{4} \mathrm{O}_{12}$ Perovskite, Nature 458, 60 (2009). 